\title{
Content Analysis of Frequency of Information About Developmental Dysplasia of the Hip on Twitter
}

\author{
Neha Kodali ${ }^{1} \cdot$ Bernita V. Sidhu ${ }^{1} \cdot$ Judy So $^{1} \cdot$ Eva Habib $^{1} \cdot$ Julie M. Robillard $^{2} \cdot$ Kishore Mulpuri $^{1,3}$. \\ Emily K. Schaeffer ${ }^{1,3}$ (1)
}

Received: 23 August 2021 / Accepted: 15 October 2021 / Published online: 2 November 2021

(C) Indian Orthopaedics Association 2021

\begin{abstract}
Introduction Early detection and screening of developmental dysplasia of the hip (DDH) are critical in reducing complications; thus, advocacy and awareness are paramount. Social media, Twitter, in particular, connects users based on their content engagement, allowing global audience to interact with one another, providing an opportunity to spread awareness and support for children and families affected by conditions such as DDH. The objective of this study was to assess the frequency and content of information being published on Twitter pertaining to DDH.

Methods A search strategy using key terms was developed. An a priori coding guide using search terms was then created to establish a framework to identify themes regarding DDH on Twitter. Data were extracted over one-week periods in June and July of 2019 and 2020. An excel-based coding guide was developed to organize and analyze the extracted data.

Results A total of 142 tweets were extracted for analysis; 41 in 2019 and 101 in 2020. The most frequently used search terms were hip dysplasia, developmental dysplasia of the hip, and dislocated hip. In 2019, the most frequent tweet authors were healthcare organizations $(13 / 41,31.7 \%)$ and healthcare professionals $(12 / 41,29.3 \%)$. In contrast in 2020, 32.7\% (33/101) tweets were authored by patients. Prevention, treatment, and general discussions were top categories in 2019, compared to education, awareness, and general discussions in 2020.

Conclusion Our data indicate that user engagement with DDH content on Twitter increased by 2.5-fold from 2019 to 2020. We found similar patterns regarding the types of authors and content type in both years. However, focus shifted toward topics of education and awareness in 2020.

Significance Our analysis identified trends and gaps in the use of Twitter that could be implemented by users to optimize messages, such as connecting directly and conversing with users through replies. Findings also indicate the importance of multi-author engagement to increase the breadth of distribution of information. Social media platforms can efficiently disseminate information to a wide range of individuals; however, they must be leveraged appropriately to accurately inform the public about DDH. Engaging with Twitter users hold great potential to promote advocacy and increase early detection and screening for DDH.

Level of evidence 3 .
\end{abstract}

Keywords Developmental Dysplasia of the Hip (DDH) · Twitter · Content analysis · User engagement

Emily K. Schaeffer

Emily.Schaeffer@cw.bc.ca

1 Department of Orthopaedic Surgery, British Columbia Children's Hospital, 1D.18 -4480 Oak St., Vancouver, BC V6H 3V4, Canada

2 Department of Medicine, University of British Columbia, Vancouver, BC, Canada

3 Department of Orthopaedics, University of British Columbia, Vancouver, BC, Canada

\section{Introduction}

Social media platforms are currently among the most accessible methods of information dissemination and have the ability to provide the public with instant access to information regarding various health conditions. The number of social media platforms and their user engagement has grown at a rapid pace over the last decade and are frequently utilized by the global population on a daily basis. Unlike traditional media, such as radio and television, social media 
platforms provide the opportunity to instantly engage and contribute to discussions [1]. Social media platforms are simple to use and accessible at most locations across the globe. As observed in 2020, the Covid-19 pandemic caused individuals across the globe to seek healthcare-related information from social media platforms [2]. This supports the great potential for social media platforms to address various health concerns on a larger scale for a global audience. Moreover, the accessibility of social media platforms may enhance access to preventative medicine, as the general public can interact with healthcare professionals and advocacy groups [3]. Twitter in particular has the ability to attract a global audience. The interactive features of Twitter, in conjunction with its limitations of 280 characters per Tweet, combine to allow for precise and condensed communication. Twitter currently functions as one of the most robust places to publish information and obtain knowledge, seeing a daily stream of 400 million tweets [4]. Choo et al. [5] discussed the use of Twitter as a medium of communication and knowledge exchange in academic medicine and highlighted that it serves as one of the most popular social media platforms that healthcare providers use to share scientific information with the public [5]. Examining the efficacy of social media platforms for the dissemination of healthrelated information requires assessing the current frequency and content of published health-related information. Tougas et al. [6] examined user engagement of social media posts pertaining to children's health and indicated that social media can increase engagement and communication with the public regarding health information, particularly when evidence-based content is published [6]. The objective of this study is to analyze the content and the frequency of information disseminated on Twitter concerning developmental dysplasia of the hip (DDH). DDH encompasses a wide spectrum of pediatric hip conditions that occur primarily among infants, caused by irregularities in the hip joint. The misalignment of the femoral head and the acetabulum can cause significant complications throughout the child's life, particularly if diagnosed late. Early detection, ideally within the first three months, and screening of infants with risk factors for hip dysplasia are paramount to reduce the occurrence of future complications [7]. Common risk factors for DDH include breech position during gestation and birth, family history of the condition, and history of clinical instability [8]. Incorrect swaddling in infancy may also be associated with DDH $[9,10]$. Through advocacy and education of DDH, the likelihood of infants being screened for hip dysplasia increases, thereby reducing future complications.

This study aims to gain insight regarding the quality of information that is available to the public about DDH on Twitter, the types of people and organizations that publish the posts, and the primary purpose of the posts. Our analysis may aid in the identification of gaps in the usage of Twitter to optimize user engagement regarding DDH content to enhance awareness and advocacy efforts.

\section{Methods}

All data collected from Twitter.com was available to the public and did not include any tweets from private accounts. Cache and cookies were cleared ahead of time to prevent information bubbles, and delays in viewing and accessing Tweets. Data was extracted using the following search terms in relation to DDH: Pavlik, Pavlik harness, Rhino cruiser, Abduction brace, DDH, Developmental dysplasia of the hip, Hip dysplasia, Dislocated hip, Dega osteotomy, Pemberton osteotomy, Adult hip dysplasia, Periacetabular osteotomy, and Acetabular dysplasia. The advanced search function on Twitter was utilized to isolate the search terms individually. Data extracted included: date of the post, type of post, type of author, source, search terms, copy of the post, content type, and additional links.

Data was collected for two separate weeks in 2019 and the same respective two weeks in 2020. Dates were chosen to include one week during Hip Dysplasia Awareness Month (June 23-30, 2019 and 2020), and one week outside of Hip Dysplasia Awareness Month (July 14-21, 2019 and 2020) each year. Dates were chosen accordingly to account for Hip Dysplasia Awareness Month and assess if this resulted in more Tweets being produced during this period in comparison to Tweets that were produced during the one week outside of Hip Dysplasia Awareness Month.

We constructed an a priori coding guide to identify the themes of hip dysplasia content discussed on Twitter. The methodology for the coding guide was constructed based on previous social media studies to identify which themes were frequently discussed [11]. Robillard et al. [11] empirically examined health information on Twitter and identified primary users, such as healthcare professional, health information, and commercial, as well as core themes, such as prevention, prediction, diagnosis, and treatment [11]. We applied a similar approach while developing our coding guide pertaining to DDH content, to ensure that our data was categorized appropriately. Content-type of each extracted post was categorized into one of six main themes: awareness, education, prevention, diagnosis, treatment, and general. Each Twitter post was categorized based on the author into journal (journalists, publication services, news outlets), parent support groups, advocacy groups, healthcare organizations, and healthcare professionals. Posts were excluded from this analysis if they discussed animals with DDH, were reposts of users' Tweets, were posts published in languages other than English, or were duplicate Tweets. Two reviewers independently coded an overlapping subset of 25 tweets, showing complete agreement. 


\section{Results}

\section{Sample Size}

A total of 142 tweets were collected over the four collection periods. In 2019, 41 tweets were collected, with 24 from June 23-30, 2019, and 17 from July 14-21, 2019.

In 2020, 101 tweets were collected, with 24 from June 23-30, 2020 and 77 from July 14-21, 2020. In the July 14-21, 2020 period, 17 reply tweets containing a keyword were collected in addition to tweets containing a keyword.

\section{Search Term Use}

In 2019, the most popular search terms were hip dysplasia $(21 / 41,51.2 \%)$ and developmental dysplasia of the hip (7/41, $17.1 \%)$. In comparison, hip dysplasia (47/101, 46.5\%), dislocated hip (16/101, 15.8\%), and developmental dysplasia of the hip $(15 / 101,14.9 \%)$ were the most popular search terms in 2020. There were no tweets containing the search terms Rhino cruiser, Abduction brace, Dega osteotomy, or Pemberton osteotomy in either year. The breakdown of the frequency of all search terms is shown in Table 1.

\section{Types of Authors Engaged on Twitter}

In 2019, healthcare organizations contributed the largest number of tweets $(31.7 \%, 13 / 41)$ and healthcare professionals $(29.3 \%, 12 / 41)$. In 2020 , the most frequent tweets were published by the general public/patients $(32.7 \%, 33 / 101)$, followed by healthcare organizations and healthcare professionals $(14.9 \%, 15 / 101)$ (Table 2).

\section{Identification of Content Type}

In 2019, Tweets which discussed prevention were most prevalent $(31.7 \%, 13 / 41)$, followed by Tweets that discussed

Table 1 Percentage of Tweets based on Search terms in 2019 and 2020

\begin{tabular}{lcc}
\hline Search & 2019 & 2020 \\
\hline Acetabular dysplasia & $4.9 \%(2 / 41)$ & $2 \%(2 / 101)$ \\
Adult hip dysplasia & $2.4 \%(1 / 41)$ & $2 \%(2 / 101)$ \\
DDH & $7.3 \%(3 / 41)$ & $8.9 \%(9 / 101)$ \\
Developmental dysplasia of the hip & $17.1 \%(7 / 41)$ & $14.9 \%(15 / 101)$ \\
Dislocated hip & $9.8 \%(4 / 41)$ & $15.8 \%(16 / 101)$ \\
Hip dysplasia & $51.2 \%(21 / 41)$ & $46.5 \%(47 / 101)$ \\
Pavlik & $2.4 \%(1 / 41)$ & $2 \%(2 / 101)$ \\
Pavlik harness & & $5.9 \%(6 / 101)$ \\
Periacetabular osteotomy & $4.9 \%(2 / 41)$ & $2 \%(2 / 101)$ \\
\hline
\end{tabular}

Table 2 Percentage of Tweets based on type of author in 2019 and 2020

\begin{tabular}{lll}
\hline Authors & 2019 & 2020 \\
\hline Advocacy groups & $12.2 \%(5 / 41)$ & $7.9 \%(8 / 101)$ \\
Parent advocacy & $17.1 \%(7 / 41)$ & $12.9 \%(13 / 101)$ \\
General public/patients & $2.4 \%(1 / 41)$ & $32.7 \%(33 / 101)$ \\
Healthcare professional & $29.3 \%(12 / 41)$ & $14.9 \%(15 / 101)$ \\
Healthcare organization & $31.7 \%(13 / 41)$ & $19.8 \%(20 / 101)$ \\
$\begin{array}{l}\text { Publication (journal publication, } \\
\text { news outlet, legal services) }\end{array}$ & $7.3 \%(3 / 41)$ & $11.9 \%(12 / 101)$ \\
\hline
\end{tabular}

treatment $(26.8 \%, 11 / 41)$. In 2020, Tweets which distributed information on education $(32.7 \%, 33 / 101)$ and Tweets associated with awareness $(29.7 \%, 30 / 101)$ were most popular (Table 3).

\section{Use of External Links in Tweets}

In $2019,11 / 41(26.8 \%)$ of the published Tweets contained an external link in the text body. All tweets containing an external link were published by publication services, healthcare professionals, healthcare organizations, and advocacy groups. In 2020, our analysis indicated that 34/101 (33.7\%) of Tweets included an external link in the text body. All Tweets by publication services and healthcare organizations contained a link associated with the Tweet.

\section{Discussion}

In the span of one year, user engagement with DDH content increased by $146.3 \%$. Despite the marked increase in user engagement, the limited pool of Tweets suggests that currently, this is an area of untapped potential to promote awareness and disseminate DDH information on Twitter. Our recommendations for increasing user engagement are described in Table 4. Our data highlighted that healthcare organizations published the largest number of general DDH-related Tweets, which could suggest that healthcare

Table 3 Percentage of Tweets based on content type in 2019 and 2020

\begin{tabular}{lcl}
\hline Content & 2019 & 2020 \\
\hline General & $17.1 \%(7 / 41)$ & $16.8 \%(17 / 101)$ \\
Education & $2.4 \%(1 / 41)$ & $32.7 \%(33 / 101)$ \\
Awareness & $9.8 \%(4 / 41)$ & $29.7 \%(30 / 101)$ \\
Prevention & $31.7 \%(13 / 41)$ & $9.9 \%(10 / 101)$ \\
Diagnosis & $12.2 \%(5 / 41)$ & $3.0 \%(3 / 101)$ \\
Treatment & $26.8 \%(11 / 41)$ & $7.9 \%(8 / 101)$ \\
\hline
\end{tabular}


Table 4 Recommendations for strengthening user engagement on social media platforms, such as Twitter
Content

Audience

Authors
Utilizing Twitter's features such as keywords, retweets, and favorites

Organizations should aim to provide users with organizationalrelated content in conjunction with personal health-related content to address the relevance of that information to the users' personal health

Multi-author engagement by healthcare professionals and healthcare organizations, would provide the public with credible information and prevent spread of inaccurate information. Multi-author engagement may also be more inclusive and could address the needs of diverse users organizations may have aimed to direct attention towards awareness and education of DDH. In contrast, healthcare professionals discussed DDH in greater detail through the primary lens of prevention and treatment methods of DDH. As a result, the difference in healthcare organizations' and healthcare professionals' utilization of Twitter highlights the need for various types of authors to publish and interact with tweets to share multifaceted knowledge of DDH on Twitter.

Hip dysplasia proved to be the most well-received search term, appearing frequently in Tweets from both 2019 and 2020. This term was utilized by all types of users, suggesting that the term 'Hip Dysplasia' is well-recognized on Twitter. In contrast, fewer tweets were observed for specific DDHrelated search terms such as Pavlik, Adult Hip Dysplasia, and Acetabular Dysplasia, and no tweets were found for medical treatment terms such as Rhino Cruiser, Abduction Brace, Dega Osteotomy, and Pemberton Osteotomy. In 2019, increased user engagement with DDH content was identified during the month of June, which may be attributed to June being International Hip Dysplasia Awareness month. However, we observed increased user engagement in July compared to June in 2020, indicating the need to spread International Hip Dysplasia Awareness month further to allow Twitter to serve as a tool in raising awareness.

One of Twitter's major strengths is its ability to create interactive chains among users through reply tweets. Our findings indicated that in 2020, the general public/patients were the primary authors of DDH posts on Twitter, and all extracted reply Tweets were also by this group. Identifying and replying to Tweets that consist of keywords could serve as a bridge for healthcare organizations to engage with individuals exploring DDH. For example, a parent tagged a healthcare organization in their Tweet, and the organization was able to find this Tweet and reply [4]. This interaction created an open dialogue and helped the organization further circulate its mission and interests. User mentions and reply tweets are a few of Twitter's features that allow healthcare organizations to reach a large audience. Through the increased development of integrated Twitter analysis tools, organizations may utilize the platform's features to reach those that are interested in DDH who might not have otherwise sought help, including through the use of external links.
Current literature discussing the strengths of Twitter's interactive features provides a deeper understanding regarding healthcare organizations' utilization of Twitter. Park and colleagues [12] analyzed healthcare organizations' usage of Twitter through a content analysis and identified that increased user engagement of a particular healthcare issue was observed when retweets and favorites were utilized [12]. Their findings indicated that although organizations primarily post original content, they did not utilize the retweet and reply functions as frequently, which may have impacted their user engagement [13]. Additionally, organizations demonstrated a tendency to publish Tweets about organization-related topics, rather than personal health-related tweets, which comprised of a small portion of the Tweets in their sample [14]. However, followers indicated to have a higher likelihood of retweeting personal health-related Tweets [14]. Therefore, healthcare organizations and advocacy groups may benefit from publishing content related to the organization's mission and interests, as well as publish personal health-related content to increase user engagement and garner the attention of a larger audience on social media platforms.

Publication services and journals primarily utilized Twitter as an additional platform to share academic papers regarding DDH. Furthermore, Choo et al. [5] also stated that Twitter can serve as a cross-disciplinary medium for disagreement, differing opinions, as well as praise of academic papers $[5,14]$. Due to the dominating social presence of Twitter, published Tweets are essentially unchecked vessels of knowledge and may contribute to a widespread distribution of false information; therefore, information published on Twitter should be approached with caution [13]. Social media platforms are currently used as one of the most common methods for information dissemination to a diverse global audience; therefore, it may be beneficial for healthcare organizations, healthcare professionals, and advocacy groups to filter through published information to assess the accuracy and ensure appropriate information is provided to Twitter users. Given Twitter's prominent role in the daily spread of information, further research assessing the accuracy of tweets containing information regarding $\mathrm{DDH}$ would validate 
and support the use of Twitter for the dissemination of DDH-related content.

The analysis of our findings indicated gaps in user engagement between healthcare professionals, healthcare organizations, and the general public. Multi-author engagement on Twitter would address and discredit inaccurate information by providing the general public with credible information, which could strengthen DDH information disseminated on Twitter. The primary limitation of our study included was the small sample of Tweets analyzed. This limited pool of Tweets may have impacted our analysis, as we were unable to accurately identify whether healthcare organizations and healthcare professionals are reaching out and providing support to patients/families and those most impacted by DDH. An additional limitation is that only one social media platform has been examined. Examining all social media platforms was beyond the scope of this study; however, we plan to look similarly across other social media platforms in the future. Twitter was chosen as it is utilized by a diverse audience, and has quickly become popular, particularly amongst healthcare providers, as a medium of scientific and knowledge exchange [5]. In future, it will be insightful to compare the scope and type of information and engaged audience across other social media platforms.

\section{Future Considerations}

Healthcare organizations and advocacy groups should aim to engage with the public using a strategic method, by providing a wide range of content and using key terms for appropriate identification within each post. As observed, social media platforms hold great potential in increasing awareness for DDH. We aim to further monitor trends associated with the use of social media for the advocacy of DDH and implement tools to provide the public with credible resources to increase awareness of DDH and promote early detection and screening.

Funding No funds were received directly for this study. Kishore Mulpuri receives research funding support from the I'm a HIPpy Foundation, The Peterson Fund for Global Hip Health, the Pediatric Society of North America (POSNA), the International Hip Dysplasia Institute (IHDI), BC Children's Hospital Foundation, the Canadian Orthopaedic Foundation, Divi's Foundation for Gifted Children, Orthopediatrics, Pega Medical and Johnson \& Johnson.

\section{Declarations}

Conflict of interest The authors declare no conflicts of interest for this study.

Ethical approval This article does not contain any studies with human or animal subjects performed by the any of the authors.
Informed consent For this type of study informed consent is not required.

\section{References}

1. Norman, C. D. (2012). Social media and health promotion. Global Health Promotion, 19(4), 3-6.

2. Zhao, X., Fan, J., Basnyat, I., \& Hu, B. (2020). Online health information seeking using "\#COVID-19 patient seeking help" on Weibo in Wuhan, China: descriptive study. Journal of Medical Internet Research, 22(10), 22910.

3. Levac, J. J., \& O'Sullivan, T. (2010). Social media and its use in health promotion. Interdisciplinary Student Journal of Health Sciences, 1(1), 47.

4. Zubiaga, A., Spina, D., Martínez, R., \& Fresno, V. (2014). Realtime classification of Twitter trends. Journal of the Association for Information Science and Technology, 66(3), 462-473. https:// doi.org/10.1002/asi.23186

5. Choo, E., Ranney, M., Chan, T., et al. (2014). Twitter as a tool for communication and knowledge exchange in academic medicine: A guide for skeptics and novices. Medical Teacher, 37(5), 411-416. https://doi.org/10.3109/0142159x.2014.993371

6. Tougas, M. E., Chambers, C. T., Corkum, P., Robillard, J. M., Gruzd, A., Howard, V., \& Hundert, A. S. (2018). Social media content about children's pain and sleep: Content and network analysis. JMIR Pediatrics and Parenting, 1(2), e11193.

7. Infant diagnosis-international hip dysplasia institute. Hipdysplasia.org. Published April 27, 2020. https://hipdysplasia.org/infantchild/infant-diagnosis/. Accessed 28 Jan 2021

8. Mulpuri, K., \& Song, K. M. (2015). AAOS clinical practice guideline: detection and nonoperative management of pediatric developmental dysplasia of the hip in infants up to six months of age. Journal of American Academy of Orthopaedic Surgeons, 23(3), 206-207.

9. Sharpe, P., Mulpuri, K., Chan, A., \& Cundy, P. J. (2006). Differences in risk factors between early and late diagnosed developmental dysplasia of the hip. Archives of Disease in Childhood Fetal and Neonatal Edition, 91(3), F158-F162.

10. Hip-healthy swaddling - international hip dysplasia institute. Hipdysplasia.org. Published May 28, 2020. https://hipdysplasia.org/ infant-child/hip-healthy-swaddling/. Accessed 28 Jan 2021

11. Robillard, J. M., Johnson, T. W., Hennessey, C., Beattie, B. L., \& Illes, J. (2013). Aging 2.0: health information about dementia on Twitter. PLoS ONE, 8(7), e69861.

12. Park, H., Reber, B., \& Chon, M. (2015). Tweeting as health communication: Health organizations' use of Twitter for health promotion and public engagement. Journal of Health Communication, 21(2), 188-198. https://doi.org/10.1080/10810730.2015. 1058435

13. Kortum, P., Edwards, C., \& Richards-Kortum, R. (2008). The impact of inaccurate Internet health information in a secondary school learning environment. Journal of Medical Internet Research, 10(2), e17. https://doi.org/10.2196/jmir.986

14. Mohammadi, E., Thelwall, M., Kwasny, M., \& Holmes, K. (2018). Academic information on Twitter: A user survey. PLoS ONE, 13(5), 0197265.

Publisher's Note Springer Nature remains neutral with regard to jurisdictional claims in published maps and institutional affiliations. 